\title{
Identification of in vivo Sulci on the External Surface of Eight Adult Chimpanzee Brains: Implications for Interpreting Early Hominin Endocasts
}

\author{
Dean Falk ${ }^{\mathrm{a}, \mathrm{b}}$ Christoph P.E. Zollikofer ${ }^{\mathrm{c}}$ Marcia Ponce de León ${ }^{\mathrm{c}}$ \\ Katerina Semendeferid José Luis Alatorre Warren ${ }^{c}$ William D. Hopkins ${ }^{e, f}$ \\ ${ }^{\text {a }}$ Department of Anthropology, Florida State University, Tallahassee, FL, USA; b School for Advanced Research, \\ Santa Fe, NM, USA; ${ }^{C}$ Anthropological Institute, University of Zurich, Zurich, Switzerland; ${ }^{d}$ Department of

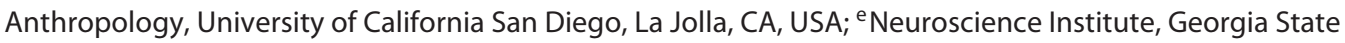 \\ University, Atlanta, GA, USA; ${ }^{f}$ Division of Developmental and Cognitive Neuroscience, Yerkes National Primate \\ Research Center, Atlanta, GA, USA
}

\section{Keywords}

Annectant gyri · Arcuate fasciculus - Australopithecines ·

Broca's area · Chimpanzee brains · Cortical sulci · Hominin endocasts · Language evolution · Lunate sulcus

\section{Abstract \\ The only direct source of information about hominin brain evolution comes from the fossil record of endocranial casts (endocasts) that reproduce details of the external morphol- ogy of the brain imprinted on the walls of the braincase dur- ing life. Surface traces of sulci that separate the brain's con- volutions (gyri) are reproduced sporadically on early hom- inin endocasts. Paleoneurologists rely heavily on published descriptions of sulci on brains of great apes, especially chim- panzees (humans' phylogenetically closest living relatives), to guide their identifications of sulci on ape-sized hominin endocasts. However, the few comprehensive descriptions of cortical sulci published for chimpanzees usually relied on post mortem brains, (now) antiquated terminology for some sulci, and photographs or line drawings from limited per-}

\section{KARGER}

(C) 2018 S. Karger AG, Basel

E-Mail karger@karger.com

www.karger.com/bbe spectives (typically right or left lateral views). The shortage of adequate descriptions of chimpanzee sulcal patterns partly explains why the identities of certain sulci on australopithecine endocasts (e.g., the inferior frontal and middle frontal sulci) have been controversial. Here, we provide images of lateral and dorsal surfaces of 16 hemispheres from 4 male and 4 female adult chimpanzee brains that were obtained using in vivo magnetic resonance imaging. Sulci on the exposed surfaces of the frontal, parietal, temporal, and occipital lobes are identified on the images based on their locations, positions relative to each other, and homologies known from comparative studies of cytoarchitecture in primates. These images and sulcal identifications exceed the quantity and quality of previously published illustrations of chimpanzee brains with comprehensively labeled sulci and, thus, provide a larger number of examples for identifying sulci on hominin endocasts than hitherto available. Our findings, even in a small sample like the present one, overturn published claims that australopithecine endocasts reproduce derived configurations of certain sulci in their frontal lobes that never appear on chimpanzee brains. The sulcal
Dean Falk

School for Advanced Research 660 Garcia St.

Santa Fe, NM 87505 (USA)

E-Mail dfalk@fsu.edu or falk@ sarsf.org 
patterns in these new images also suggest that changes in two gyri that bridge between the parietal and occipital lobes may have contributed to cortical reorganization in early hominins. It is our hope that these labeled in vivo chimpanzee brains will assist future researchers in identifying sulci on hominin endocasts, which is a necessary first step in the quest to learn how and when the external morphology of the human cerebral cortex evolved from apelike precursors.

(c) 2018 S. Karger AG, Base

\section{Introduction}

Two major trends characterized hominin brain evolution: brains enlarged over time and their circuitry and cellular composition became more complex (neurological reorganization). Estimates of brain sizes are obtained relatively easily by measuring the volumes of braincases (cranial capacities). Research on neurological reorganization is more challenging because it entails analyzing subtle details of gyral bulges and sulci that became imprinted on the inner walls of braincases when the animals were still alive [Jerison, 1973]. These impressions are reproduced on internal casts of braincases (endocasts). Endocasts sometimes form naturally after animals die, or they may be prepared manually by casting empty skulls or constructed virtually from CT scans of fossilized skulls [Falk, 2004].

The quality of cortical imprints on endocasts varies with species (smaller-brained species within lineages yield more detailed endocasts than their larger-brained relatives [Radinsky, 1972]), developmental age (endocasts from juveniles generally reproduce more details than those from mature individuals) [Connolly, 1950; Minh and Hamada, 2017], and (in the case of fossils) geological conditions that affect preservation. The fidelity of sulcal reproduction on endocasts varies with region of the brain [Minh and Hamada, 2017], as illustrated by a study that compared traces of sulci reproduced on endocasts from six chimpanzees with the sulci on corresponding brains and found that some sulci (e.g., the fronto-orbital sulcus; fo) reproduced well, while others (e.g., the lunate; L) did not [Clark et al., 1936].

There are currently two prevailing hypotheses about the mechanisms that govern the formation of sulci during fetal development. The axon tension hypothesis posits that species-specific sulcal patterns are caused by tension along axons in subcortical white matter [Van Essen, 1997]. The mechanical folding model, on the other hand, suggests that tangential expansion of the cerebral cortex generates compression that leads to the development of cortical sulci [Tallinen et al., 2016]. A recent study of developing cortex in rhesus monkey fetuses suggests that maturation of dendrites within the cortex may be linked to the formation of sulci, which may be more consistent with the mechanical folding model [Wang et al., 2017]. Although these discussions about the mechanisms of how sulci form during development may seem somewhat tangential to the present paper, the shape of the developing brain (endocast) modulates the mechanical factors that influence the placement and orientations of sulci so that, in real brains, the "primary convolutions are consistently reproducible in their location" [Tallinen et al., 2016, p. 591]. It is important to note, however, that the only information about sulci that can be gleaned from primate, including hominin, endocasts depends on superficial traces [Pearce et al., 2013], which are often fragmentary.

Because chimpanzees are the closest living phylogenetic relatives of humans, descriptions of their brains have traditionally been important for identifying sulci on australopithecine endocasts [Dart, 1925; Clark et al., 1936; Falk, 2009], and comparison of chimpanzee and human brains remains essential for addressing brain evolution in hominins since the two groups split from a common ancestor [Semendeferi et al., 1997, 2011; Gómez-Robles et al., 2015]. The literature on chimpanzee brains prior to and during the first half of the 20th century focused mostly on individual brains [e.g., Benham and Oxoii, 1895], although a few studies included relatively large samples of chimpanzees [reviewed in Walker and Fulton, 1936]. Early authors relied exclusively on post mortem brains, used terminology that is now outdated, failed to recognize important sulci such as the middle frontal $(f m)$ sulcus and Affenspalte (lunate sulcus, $L$ ), and illustrated specimens with photographs or line drawings from limited perspectives (typically right or left lateral views) [e.g., Mingazzini, 1928; Walker and Fulton, 1936]. The important monograph of Bailey et al. [1950], i.e., "The Isocortex of the Chimpanzee," was an improvement over earlier reports because it emphasized that sulcal homologies should be based on their approximate relations to cytoarchitectural areas, and used modern terminology and correct identifications for sulci. Although Bailey et al. [1950, pp. 18-19, Fig. $4 \mathrm{a}-\mathrm{c}$ ] provided line drawings that illustrated and labeled the sulci for one generic left chimpanzee hemisphere, they did not include illustrations of individual chimpanzee brains with comprehensively labeled sulci.

John Connolly [1950] advanced knowledge of chimpanzee (and other primate) sulci in his seminal monograph, "External Morphology of the Primate Brain." His
Falk/Zollikofer/Ponce de León/ Semendeferi/Alatorre Warren/Hopkins 
identifications of homologous primate sulci relied heavily on comparisons of brains across the primate order in light of cytoarchitectonic and developmental evidence as well as examinations of occasional submerged (buried) gyri and sulci, and his sulcal identifications have stood the test of time. For example, a recent study using high-resolution imaging and semiautomatic detection of sulci on 46 virtual endocasts representing 11 genera of Old World monkeys [Beaudet et al., 2016] identified sulci that were consistent with the descriptions of Connolly [1950] of cercopithecoid brains and the identifications of Falk [1978] of sulci reproduced on cercopithecoid endocasts. Importantly, Connolly [1950] used modern terms for sulci (Table 1), studied the reproduction of sulci on great ape endocasts, and applied his knowledge of sulci on ape brains to interpretations of early hominin endocasts.

\section{Current Knowledge about the Main Differences between Chimpanzee and Human Sulcal Patterns}

Thanks to the research of Connolly [1950] and other early workers, it has long been known that, compared to humans, the brains of our closest "cousins," i.e., chimpanzees, manifest strikingly different sulcal patterns in two parts of the brain - the inferior frontal convolution and the region that approximates the rostral border of the lateral surface of the occipital lobes (Fig. 1). (It is worth noting that all three great ape species share the same basic sulcal pattern [Connolly, 1950].) More recent scholars have used state-of-the-art neuroimaging, cytoarchitectonic, and analytical methods to refine our understanding of these differences. Beginning with the frontal lobe, recent cytoarchitectural and topographic research on chimpanzee brains confirms that $f_{0}$, which is seen invariably in great apes, is typically located rostral to Brodmann area (BA) 44, and BA 45 is usually anterior to BA 44 [Keller, 2009; Schenker et al., 2010] (Fig. 1). Despite this general consistency, recent probabilistic mapping studies of subcortical and surface distributions of BA 44 [Schenker et al., 2010] and cytoarchitectural investigations of the subcortical and cortical extent of BA 44 [Sherwood et al., 2003] have revealed a good deal of individual variation in the precise relationship between fo and these two areas. Although some authors have mistakenly identified the subfrontal sulcus or the fronto-marginal sulcus of Wernicke (W) of humans as fo [Connolly, 1950, p. 196], a homologue of the ape fo does not occur on the surface of human brains. The homologues of BA 44 and BA 45 in left hemispheres of humans comprise Broca's speech area, and in
Table 1. Abbreviations of features identified on chimpanzee brains (sulci unless otherwise stated)

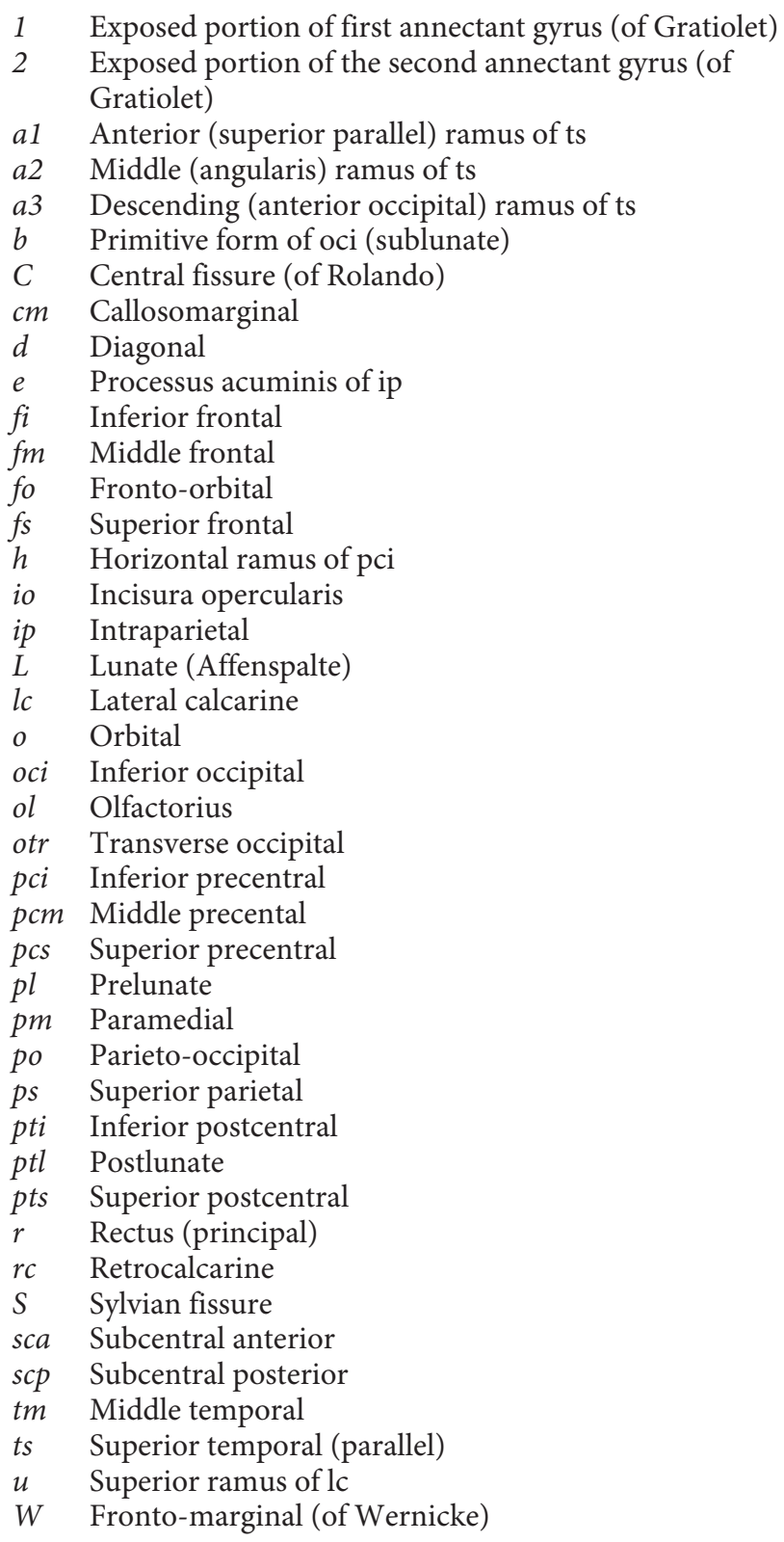

After Connolly [1950].

both hemispheres these areas are delimited partly by two recently evolved sulci, i.e., the horizontal and ascending rami of the Sylvian fissure, which define the superficial rostral and caudal borders, respectively, of the pars triangularis (BA 45). Compared to fo in great apes, the superficial relationship between these sulci and BA 44 and BA 45 is relatively precise because "there are regions, i.e., the 

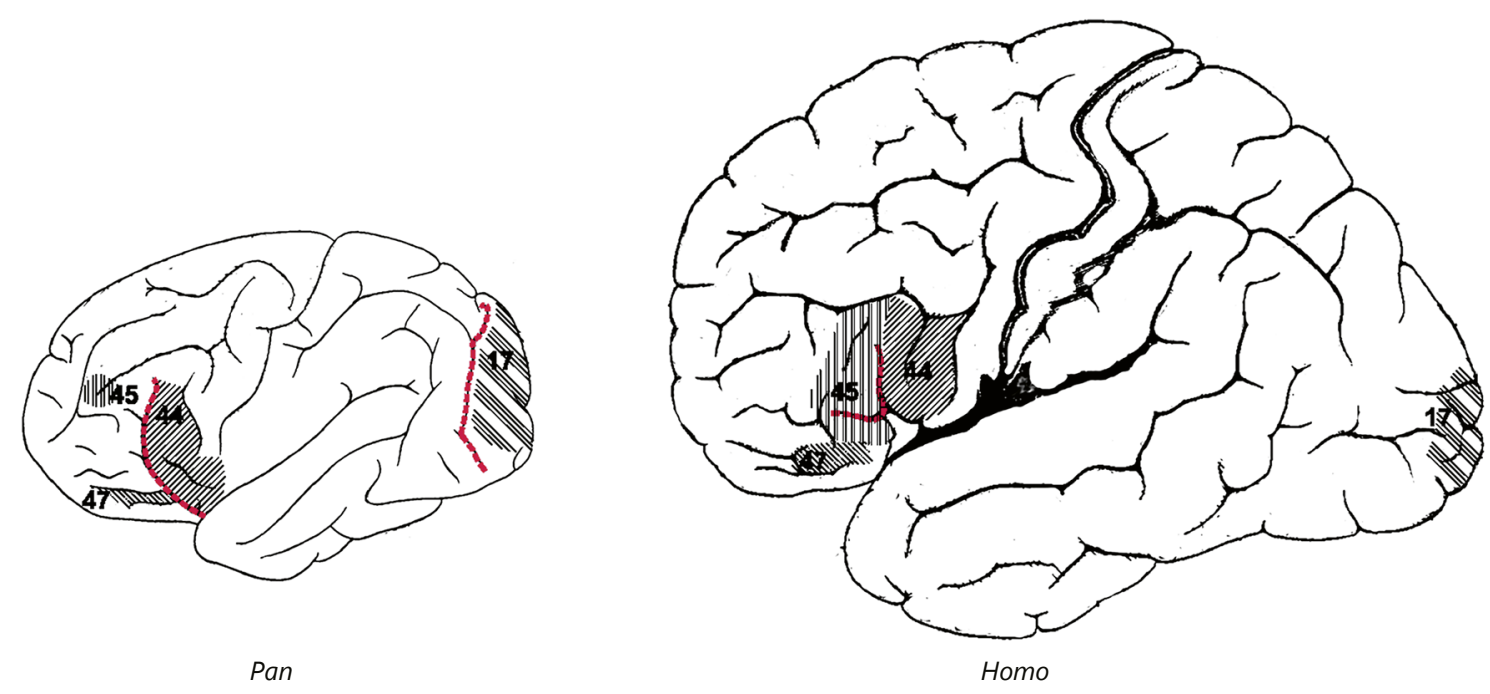

Fig. 1. Differences in sulcal patterns of chimpanzees and humans. Chimpanzee brains have two crescent-shaped sulci that are lacking in humans - the fronto-orbital sulcus approximates the anterior border of Brodmann area (BA) 44 and the lunate sulcus courses about $2 \mathrm{~mm}$ rostral to BA 17 . Unlike chimpanzee brains, the free

free surfaces of the triangular and opercular parts, in which the probability is very high of localizing areas 45 and 44, respectively" [Amunts et al., 1999, p. 339].

The above findings are consistent with the hypothesis of Connolly [1950] that, as the inferior frontal convolution expanded during the course of hominin brain evolution, the inferior part of $f o$ was displaced caudo-ventrally beneath the external surface of the brain to become the anterior limiting sulcus of the insula. In humans, this limiting sulcus, thus, represents the homologue of the inferior part of the chimpanzee fo [Connolly, 1950, p. 330]. Connolly [1950] also observed that, during human fetal development, "a wedge of cortex passes between the orbital operculum (BA 47) and the dorsal or fronto-parietal operculum (BA 44), so that two branches R and R' enclosing area 45 are formed" [Connolly, 1950, p. 330; see also p. 162, Fig. 111]. Connolly suggested that a similar process explained the evolutionary caudo-ventral migration of BA 45 and the emergence of the pars triangularis with its limiting sulci in humans, and hypothesized further that evolutionary reorganization of frontal association cortices was associated with the emergence of a phylogenetically new caudal extension of $\mathrm{fm}$ [Connolly, 1950, p. 197] as well as expansion of the orbital margin "downward and outward increasing anteriorly the breadth of the lobe" [Connolly, 1950, p. 75].

surface of BA 45 in human brains is bordered on two sides by anterior horizontal and ascending branches of the Sylvian fissure. Left hemispheres are depicted; numbers represent Brodmann areas. See Bailey et al. [1950] and Von Bonin [1949] for details. Reproduced from www.deanfalk.com.

As noted, sulcal patterns on the external surfaces of the occipital lobes also differ between chimpanzees and humans (Fig. 1). In chimpanzees a distinct crescent-shaped lunate sulcus courses transversely across the occipital lobe approximately $2 \mathrm{~mm}$ rostral to the lateral anterior border of primary visual cortex (BA 17) [Bailey et al., 1950 , p. 69]. As with the inferior frontal gyrus, contemporary researchers have also filled in some details concerning this region. In humans the lateral representation of BA 17 is greatly reduced and highly variable [Amunts et al., 2000] and representation of BA 17 may be lacking entirely from the lateral convexity of their occipital lobes - e.g., in $65 \%$ of 20 human hemispheres in one study [Rademacher et al., 1993]. In instances where BA 17 appears on the lateral surface of human brains, it is located considerably caudally compared to the representation for apes and, unlike the latter, its rostral edge is not typically bordered by a sulcus (Fig. 1) [Allen et al., 2006]. However, polar sulci sometimes appear near the rostral boundary of the human striate area on the convex surface, but these are "not interpreted...as parts of the true lunate sulcus" [Connolly, 1950, p. 232].

Efforts to understand how hominin brains evolved from having an apelike L to having occipital lobes that lack this prominent sulcus (Fig. 1) have been hampered by confusion in both the classic and contemporary compara-
Falk/Zollikofer/Ponce de León/ Semendeferi/Alatorre Warren/Hopkins 
tive literature. Compared to other regions, the human occipital lobe shows the most variable sulcal patterns on the lateral surface of the brain, which helps to explain why "the occipital sulci have received different names according to different authors, and the classic anatomy textbooks do not describe their variations and different patterns in detail" [Alves et al., 2012, p. 1014]. Fortunately, human temporo-occipital sulcal anatomy has been clarified by numerous researchers [Ono et al., 1990; Gilissen and Zilles, 1996; Iaria and Petrides, 2007; Iaria et al., 2008; Segal and Petrides, 2012], including Alves et al. [2012] who compared the descriptions and nomenclature from the literature and identified the most characteristic and consistent occipital sulcal patterns and their relationships with occipital gyri in twenty human hemispheres. Alves et al. [2012, p. 1015] recognized a lunate sulcus in humans as a "continuous transverse sulcus that crosses a substantial portion of the lateral surface of the posterior portion of the occipital lobe, just anterior to the occipital pole," which occurred in $25 \%$ of their specimens. However, in keeping with the high-resolution MRI study of Allen et al. [2006] of occipital sulci and their relationship with BA 17 in 110 adult humans, and contrary to the assertion of Grafton Elliot Smith [1903] that human brains have a true homologue of the ape Affenspalte ("ape sulcus," renamed the lunate sulcus by Smith [1903]) bordering the lateral representation of BA 17, Alves et al. [2012, pp. 1021-1022] concluded that hominin brain evolution: "led to the loss of a 'true' lunate sulcus in humans, and, when present, it is found in a posterior position to that observed in the great apes, with no correlation between the lunate sulcus and the primary visual cortex, and with absence of the primary visual cortex in the occipital convexity, where it has been replaced by secondary and tertiary visual association cortices. This evolutionary reorganization process resulted from cerebral expansion, and consequent increased number of temporoparietooccipital connections."

\section{Context and Objectives of the Present Report}

What paleoneurologists seek to learn from the hominin endocast record [Holloway et al., 2004a] are details about the evolutionary transition from apelike to humanlike sulcal patterns (i.e., from the pattern on the left to that on the right in Fig. 1). In other words, at what point and in what sequence did early hominin endocasts begin evolving sulcal patterns that differed from those of extant great apes? Because discoveries of hominins with apesized braincases that are more likely to reproduce rela- tively good detail of the external morphology of the brain than endocasts from larger-brained hominins are on the rise in Africa (Sahelanthropus, Australopithecus afarensis, $A$. sediba, and Homo naledi) and Indonesia (H. floresiensis), this is an exciting time for paleoneurologists. A crucial first step toward answering the above question is that sulci that are reproduced on hominin endocasts must be accurately identified. As in the past, however, there is still a lack of unanimity regarding the identifications of certain sulci on australopithecine endocasts, especially those in and near the important frontal and occipital regions illustrated in Figure 1. This is partly because early hominin sulci have sometimes been misidentified because neither their relationships with other sulci nor the current understanding of sulcal homologies have been taken into account [reviewed in Falk, 2014]. Equally concerning is that there are very few illustrations of chimpanzee brains in the literature which have comprehensive and accurate sulcal identifications and can, thus, serve as adequate references for identifying sulci on early hominin endocasts.

Connolly [1950] studied 9 postmortem brains and additional illustrations from other sources and provided line drawings with comprehensive sulcal identifications that were informed by knowledge of sulcal homologies and cytoarchitecture for only 5 chimpanzee hemispheres (Fig. 2). Although few in number, these line drawings provide the most accurate and comprehensive sulcal identifications, to date, on the external surfaces of chimpanzee brains and, as such, have been important for describing sulci on ape-sized endocasts of early hominins [Falk, 2014].

An objective of the present paper is to add to the literature on chimpanzee sulcal patterns by providing illustrations of in vivo brains for 16 hemispheres from 8 adults, which include comprehensive identification of sulci that are informed by the relationships and derivations of sulci relative to each other as well as the literature on sulcal homologies in higher primates. A second objective is to discuss the implications of these new images for previously published descriptions and interpretations of small-brained hominin endocasts.

\section{Materials and Methods}

In vivo magnetic resonance imaging (MRI) scans were provided by the Yerkes National Primate Research Center for 8 adult chimpanzees ( 4 females and 4 males; online suppl. Table S1; see www.karger.com/doi/10.1159/000487248 for all online suppl. material) ranging in age from 13 to 44 years at the time they were being surveyed for their annual physical examinations. Subjects were 


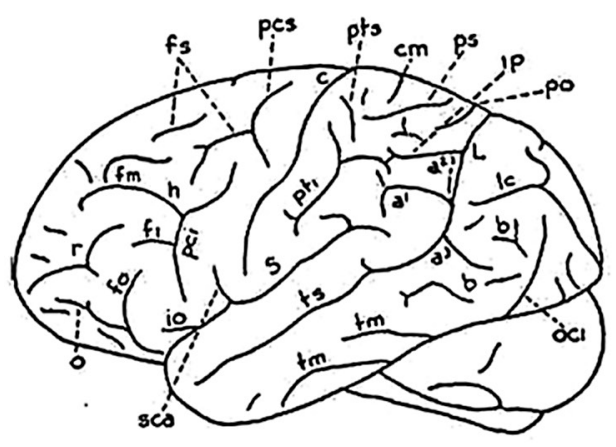

80
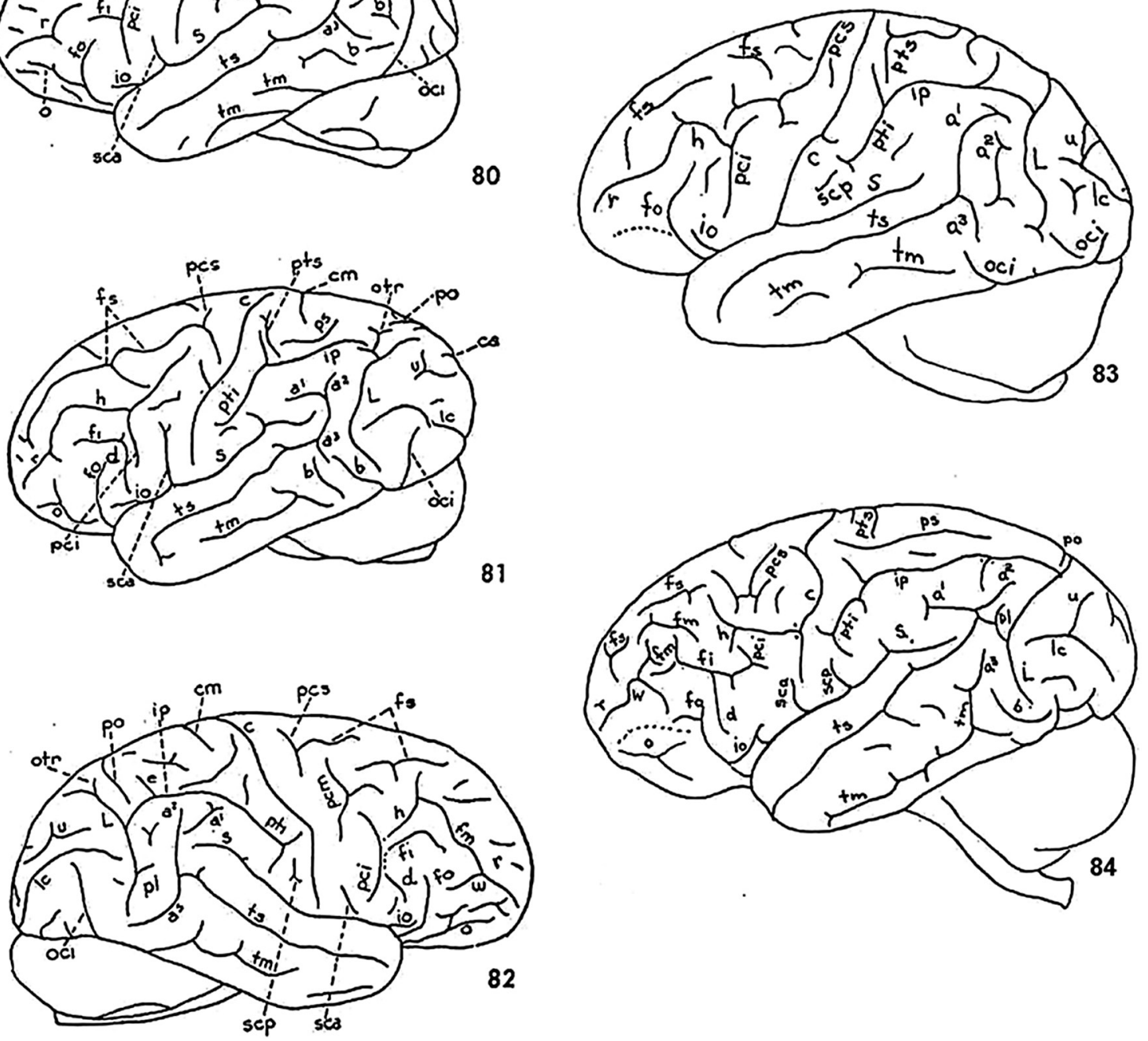

Fig. 2. Five chimpanzee hemispheres illustrated in Connolly [1950]. Abbreviations are in Table 1; images are from Connolly [1950, pp. 108, 113, 114]. Reproduced from www.deanfalk.com.

first immobilized by ketamine $(10 \mathrm{mg} / \mathrm{kg})$ or telazol $(3-5 \mathrm{mg} / \mathrm{kg})$ and subsequently anaesthetized with propofol $(40-60 \mathrm{mg} /[\mathrm{kg} / \mathrm{h}])$ following standard procedures at the Yerkes National Primate Research Center. The subjects remained anaesthetized for the duration of the scans as well as the time needed to transport them between their home cage and the imaging facility (between 5 and 10 $\mathrm{min}$ ) or mobile imaging unit (total time $\sim 5 \mathrm{~min}$ ). Subjects were placed in the scanner chamber in a supine position with their head fitted inside the human-head coil. Scan duration ranged between 40 and 60 min as a function of brain size.
Five chimpanzees (Abby, female, 13 years old; Callie, female, 15 years old; Evelyne, female, 15 years old; Joseph, male, 27 years old; and Iyk, male, 44 years) were scanned using a 3.0-T scanner (Siemens Trio; Siemens Medical Solutions USA, Inc., Malvern, PA, USA) at YNPRC. T1-weighted images were collected using a 3-dimensional gradient echo sequence (pulse repetition $=2,300 \mathrm{~ms}$, echo time $=4.4 \mathrm{~ms}$, number of signals averaged $=3$, matrix size $=$ $320 \times 320$, voxel resolution $=0.625 \times 0.625 \times 0.60 \mathrm{~mm})$. Additionally, 3 chimpanzees (Lulu, female, 44 years old; Laz, male, 20 years old; and J. Carter, male, 24 years old) were scanned using a 1.5-T
50

Brain Behav Evol 2018;91:45-58 DOI: $10.1159 / 000487248$
Falk/Zollikofer/Ponce de León/

Semendeferi/Alatorre Warren/Hopkins 


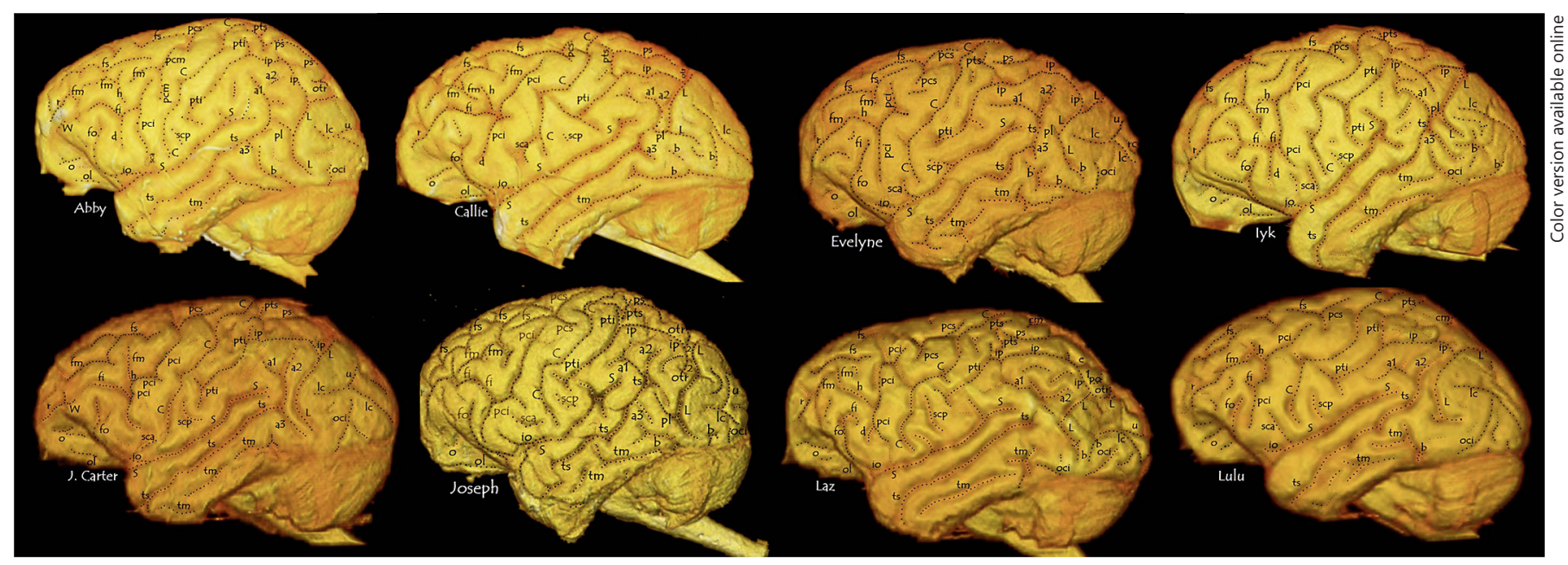

Fig. 3. Left lateral views of 8 chimpanzee brains. See Table 1 for abbreviations.

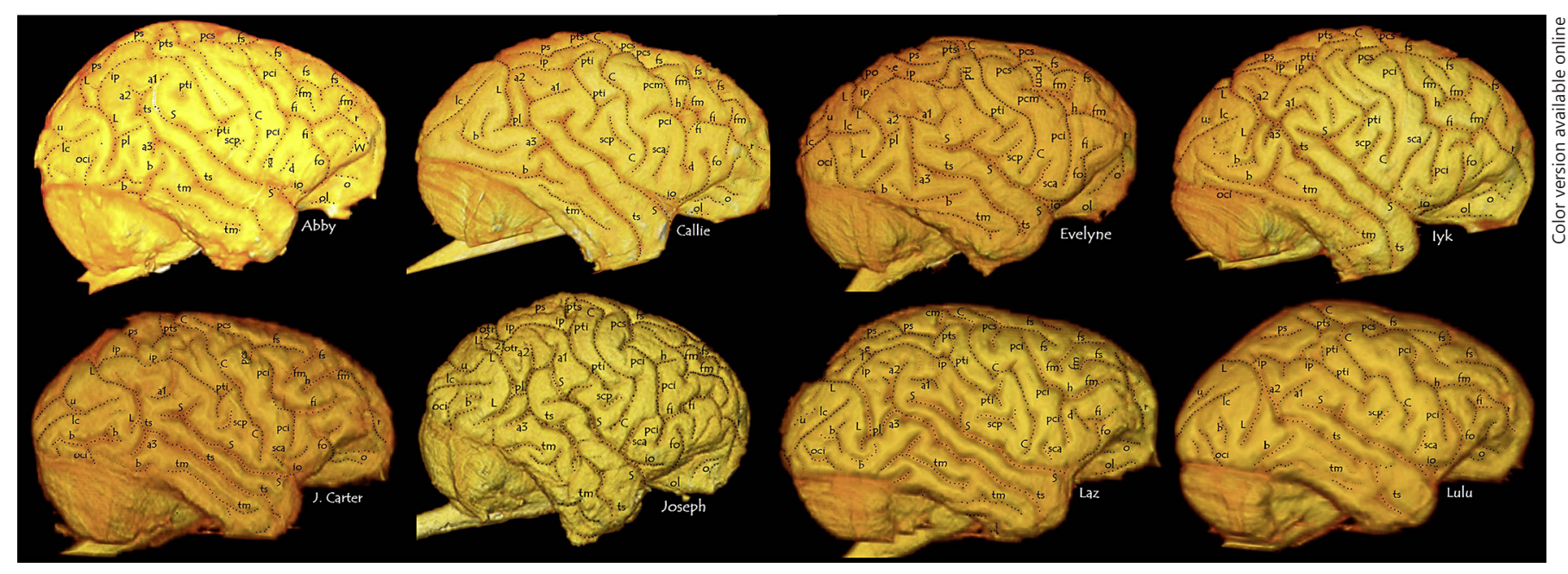

Fig. 4. Right lateral views of 8 chimpanzee brains. See Table 1 for abbreviations.

Phillips machine (The Netherlands). T1-weighted images were collected in the transverse plane using a gradient echo protocol (pulse repetition $=19.0 \mathrm{~ms}$, echo time $=8.5 \mathrm{~ms}$, number of signals averaged $=8$, matrix size $=256 \times 256$, voxel resolution $=\sim 0.78 \times$ $0.78 \times 0.70 \mathrm{~mm}$ but varied slightly across these 3 subjects). After completing MRI procedures, the subjects were temporarily housed in a single enclosure for 6-12 h to allow the effects of the anesthesia to wear off, after which they were returned to their social group.

Prior to segmentation, 3-D rendering, and sulci analysis, the MRI scans were skull-stripped, aligned in the AC-PC axis, and resampled at $1-\mathrm{mm}^{3}$ isotropic voxels to assure uniformity in the scale of measurement across brains. An MRI nonuniformity correction was applied to the aligned T1-weighted image stacks using the N3 algorithm [Sled et al., 1998] as implemented in FireVoxel (NYU Center for Advanced Imaging Innovation and Research, New York, NY, USA), as well as the 3-D Slicer (www.slicer.org) implementation of N4ITKBiasFieldCorrection, an improved ver- sion of the original N3 algorithm [Tustison et al., 2010]. Brain segmentation was performed using the interactive, region-growing segmentation tools in Avizo v8.0. This fully supervised segmentation procedure is time consuming but avoids potential biases and slight segmentation errors that tend to occur in unsupervised (automated) techniques due to differences in scanning protocols and/ or scanners. The segmented cortical data of each individual were then volume-rendered in standard anatomical views.

To aid in sulcal identifications, illustrations and discussions of chimpanzee sulcal patterns were consulted from several classic sources [Cunningham, 1890, 1892; Von Bonin, 1949; Bailey et al., 1950 ] in addition to Connolly [1950]. Identifications of the first annectant gyrus were facilitated by discussions and illustrations in Ferrier [1886] and Gratiolet [1854]. To the extent possible, sulcal identifications for chimpanzee brains and related analyses were informed by an updated understanding of homologous sulci among catarrhine primates based on comparative neuroanatomi- 


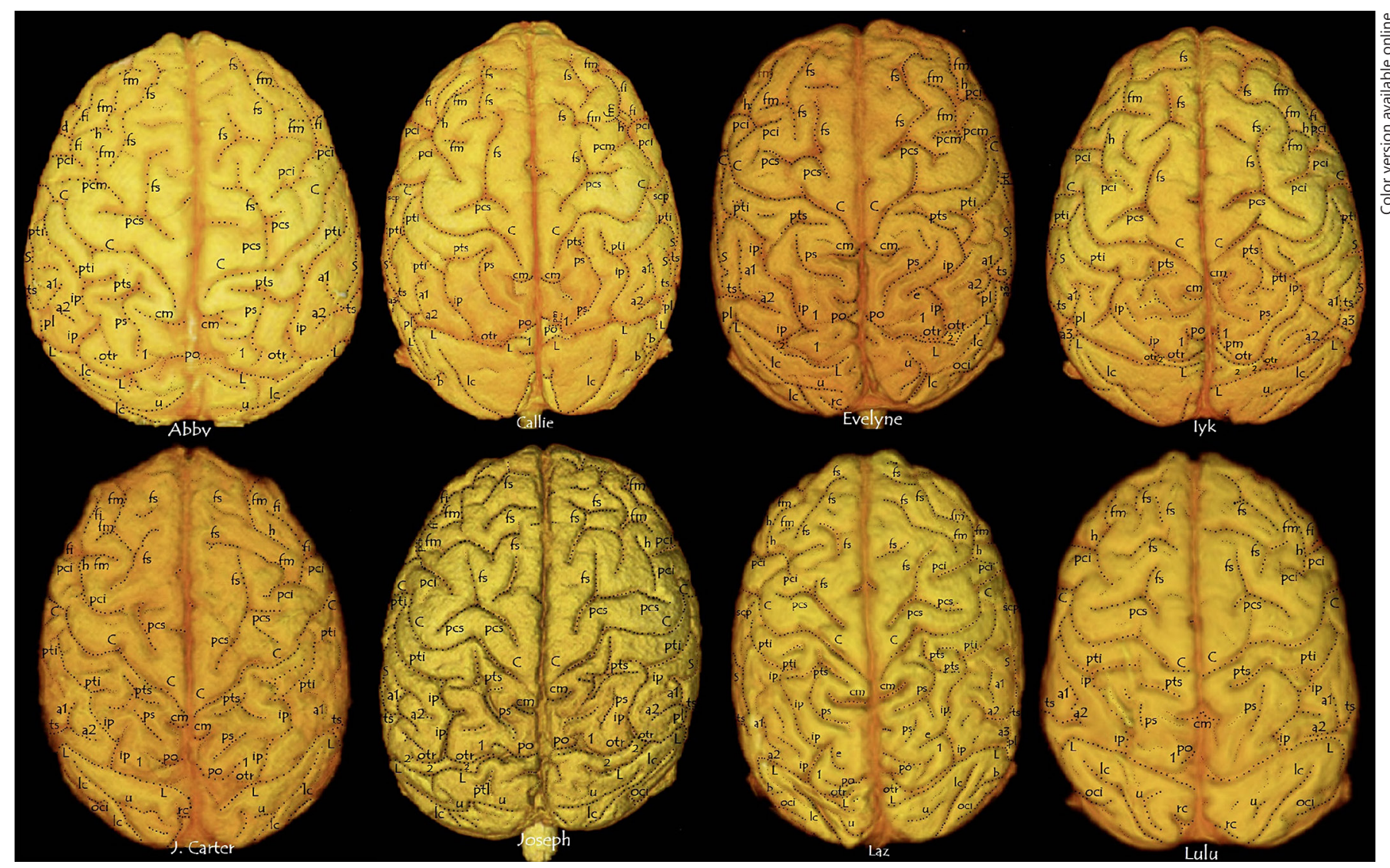

Fig. 5. Dorsal views of 8 chimpanzee brains. See Table 1 for abbreviations.

cal, cytoarchitectonic, and imaging research [Connolly, 1950; Allen et al., 2006; Iaria and Petrides, 2007; Petrides et al., 2012]. In addition to materials from the literature, partial brain casts from 2 chimpanzees and 1 gorilla were used as hard-copy 3-D references.

Cortical sulci were marked with dots and labeled on dorsal, right, and left lateral views for each chimpanzee brain (by D.F.) in Microsoft Paint using the terminology of Connolly [1950] (Table 1) in the following order: left lateral view, left side of the dorsal view, right lateral view, and right side of the dorsal view. For each brain, identifications of sulci in the lateral views were considered in light of sulcal identifications on the same side in the corresponding dorsal views and vice versa. Care was taken to distinguish sulci from blood vessels and to recognize instances in which different named sulci appeared to merge superficially.

\section{Results}

Sulci are identified on the left lateral, right lateral, and dorsal views of in vivo 3-D MRI virtual images of 8 chimpanzee brains in Figures 3-5, respectively. (Readers are encouraged to enlarge Fig. 3-5 to see the sulci more clear- ly and to make comparisons between different brains.) The three views are also illustrated for individual chimpanzee brains in online supplementary Figures S1-S8 which, as far as we know, constitute the first published set of illustrations with comprehensive sulcal identifications for in vivo chimpanzee brains viewed from three aspects. Although it is beyond the scope of the present paper, online supplementary Figures S1-S8 should be useful to researchers interested in intraindividual (left-right) variation in chimpanzee sulcal patterns. Table 1 and online supplementary Table S2 provide keys for the abbreviations used in the figures in the text and in online supplementary Figures S1-S8, respectively.

Because, to date, comparative paleoneurological studies of fossil hominin endocasts have focused largely on the two cortical regions that manifest distinctly different sulcal patterns in great apes and humans - i.e., the lateral prefrontal cortex and the dorsolateral occipital cortex (Fig. 1) - variations in the locations, configurations, and patterns of cortical sulci in and near these regions are discussed below for the 8 chimpanzee brains.
52

Brain Behav Evol 2018;91:45-58

DOI: $10.1159 / 000487248$
Falk/Zollikofer/Ponce de León/

Semendeferi/Alatorre Warren/Hopkins 


\section{Discussion}

\section{Lateral Prefrontal Cortex}

Although chimpanzee and other great ape brains manifest numerous primary sulci that are common to all higher primates (e.g., the Sylvian fissure [S], the central sulcus $[C]$, and the superior temporal sulcus $[t s])$, they also have secondary sulci that are not common, which can make it difficult to identify sulcal homologies. Thus, "it is better to note the sulcal pattern of a whole region before analyzing its furrows one by one" [Bailey et al., 1950, p. 25]. This point was well taken by Connolly [1950], who, for example, reasoned that the inferior frontal sulcus $(f i)$ and $f m$ that appear often, but not always, in the frontal lobes of great apes are derived compared to all other nonhuman primates (which never have these sulci) but retained as constant features in human brains. More specifically, Connolly [1950] argued persuasively that, in apes, $f i$ is derived from the lower part of the precentral inferior ( $p c i)$ sulcus and courses toward the fronto-marginal sulcus $(W)$ that stems from the rostral part of the rectus sulcus $(r)$, while $\mathrm{fm}$ derives from a higher horizontal branch $(h)$ of $p c i$ and the caudal (more vertical) part of $r$ (Fig. 2). Thus, to recognize these sulci on ape brains (and on early hominin endocasts [Falk, 2014]), one must take the entire sulcal pattern on the lateral surface of the frontal lobe into account.

Comparisons of cytoarchitectonic regions and their connecting pathways in macaque and human frontal lobes confirm that the basic plan of the prefrontal cortex of cercopithecine monkeys, humans, and (by inference) apes is the same [Connolly, 1950; Petrides et al., 2012]. Nonetheless, two phylogenetically "new" frontal lobe sulci, i.e., $f i$ and $f m$, appear in great apes and humans but not monkeys [Connolly, 1950]. Although from an evolutionary perspective it is important to compare the configurations of these sulci in early hominins with their patterns in extant chimpanzees and humans, knowledge about their variations in chimpanzees has, to date, been extremely limited. This gap is addressed below.

The Inferior Frontal Sulcus. Fi derived (evolved) from the inferior limb of the arcuate sulcus ( $p c i$ ) rather than $r$, with which it is sometimes connected in great apes and has sometimes been mistakenly homologized [Connolly, 1950, pp. 79, 100, 108, 110]. Thus, in apes "the true frontal inferior sulcus springs from the inferior precentral sulcus below the horizontal branch of the arcuate and (below the level of) the caudal end of the sulcus rectus" [Connolly, 1950, p. 119]. In both great apes and humans, $f i$ separates the inferior (third) convolution from the middle frontal gyrus above it, and the cytoarchitectural area below $f i$ differs from that above it for chimpanzees [Connolly, 1950, p. 108] and humans [Petrides et al., 2011, 2012 , p. 53]. Fi of great apes are typically relatively short sulci that stem from $p c i$ or appear independently just rostral to $p c i$, course horizontally directly above the medial end of $f o$ on the lateral surface of the frontal lobe, and terminate somewhat rostral to fo but caudal to $r$. (As discussed above, humans have a different sulcal pattern in this part of the frontal lobe.)

As illustrated in Figure 2, $f i$ stems directly from $p c i$ in 2 of the 5 chimpanzee hemispheres illustrated by Connolly [1950, pp. 108-114, Fig. 80, 84] and is independent of $p c i$ (but has a relationship with a derivative of $p c i$, the diagonal sulcus, $d$ ) in 2 others [Connolly, 1950, Fig. 81, 82]. $F i$ is not identified in a fifth chimpanzee hemisphere [Connolly, 1950, Fig. 83].

Fi shows considerably more variation in the 16 hemispheres described here than in the 5 hemispheres illustrated by Connolly [1950] (Fig. 2). Similar to the basic pattern in Figure 80 of Connolly [1950], fi stems directly from pci in 11 of the 16 hemispheres (bilaterally in Abby, Callie, Iyk, J. Carter, and Joseph, and in Lulu's left hemisphere Fig. 3,4 ), although most of these $f i$ are longer and/or have various connections with other sulci compared to the simple configuration shown in Fig. 80 of Connolly [1950]. The shortest $f i$ that is attached to $p c i$ in our sample appears in Lulu's left hemisphere, although there is at least one independent fragment of $f i$ rostral to it (Fig. 3). (The other unlabeled fragment that is rostral to Lulu's fo is likely an element of $W$ rather than fi.) Lulu's right hemisphere (Fig. 4), on the other hand, has two independent fragments of $f i$, which show a more complicated pattern than appears in any of Connolly's specimens. The shortest fragment is a dorsal extension of $f_{o}$, and rostral to it is a longer tri-radiate fragment of $f i$ that shows that the dorsal border of the inferior frontal convolution extends noticeably rostral to the so-called "orbital cap," which is delimited anteriorly by fo. Relatively elongated fis that stem from $p c i$ in the right hemispheres of Callie, J. Carter, and (especially) Joseph (Fig. 4), and the left hemispheres of Joseph and Iyk (Fig. 3) reveal that it is not unusual for chimpanzee brains to have inferior convolutions that extend considerably rostral to the orbital cap.

There are other interesting variations related to $f i$. In addition to the right hemisphere of the aforementioned Lulu, $f i$ occurs independently of $p c i$ and is attached to $f o$ in both hemispheres of Evelyne and Laz (Fig. 3, 4). A number of specimens have one or more fragments of $f i$ bordering and within their inferior frontal convolutions, which may also have diagonal sulci $(d)$ (left hemispheres 
of Callie, Evelyne, Iyk, Laz, and Lulu [Fig. 3]; right hemispheres of Abby, Callie, Iyk [notice the interesting switchback in the path of $f i]$, J. Carter, Joseph, and Lulu [Fig. 4]). The inferior frontal convolutions of these chimpanzees, thus, appear more convoluted than most of the specimens illustrated by Connolly [1950] (his Fig. 84 being a possible exception).

It is important to emphasize that fragments of $f i$ appear to extend or embellish the medial ends of $f_{0}$ in a number of specimens (left hemispheres of Callie [in particular, note the triangular patch at the medial end of $f_{0}$ ], Evelyne, Laz [Fig. 3]; right hemispheres of Abby, Evelyne, J. Carter, Laz, and Lulu [Fig. 4]). As discussed below, some of the above findings for $f i$ in chimpanzees have implications for published descriptions of early hominin endocasts.

The Middle Frontal Sulcus. As detailed by Connolly $[1950$, pp. $75-6,100,108]$, the rostral part of $f m$ in apes appears to be derived phylogenetically from the dorsal, more vertical part of $r$ (the rectus or principal sulcus, which is present in monkeys) rather than its fronto-marginal branch $(W)$. The caudal end of $f m$, on the other hand, is often connected to (derived from) the superior (horizontal) branch $(h)$ of pci (arcuate) sulcus [see also Von Bonin, 1949, p. 31; Bailey et al., 1950, p. 18; Duvernoy et al., 1999, p. 7; Petrides et al., 2011, 2012]. Connolly [1950] illustrated the following variations in $\mathrm{fm}$ for 5 chimpanzee hemispheres (Fig. 2). In his most complicated specimen, a fragment appeared within the middle frontal gyrus and a second extended from the caudal end of $r$ (84 in Fig. 2). In a second specimen, $f m$ was attached to the rostral end of $h$ (80 in Fig. 2). Fm was joined to both $r$ and $h$, creating the superficial appearance of one elongated sulcus that extended caudally and obliquely from near the frontal pole to $h$ in a third hemisphere [82 in Fig. 2]. Although Connolly [1950] did not label $\mathrm{fm}$ in two other hemispheres, he wrote that $r$ was connected with $h$ "through the medium of an element of the middle frontal sulcus" for one (81 in Fig. 2) and that for a young specimen (83 in Fig. 2) $h$ "is continuous with the s. rectus and the two constitute the midfrontal sulcus."

$F m$ in the 16 chimpanzee hemispheres shown here are located below $f s$ and above $f i$ (i.e., are in the middle frontal gyrus, although fragments of $f m$ sometimes intersect $f s$ and $f i$ ) (Fig. 3, 4). All of the variations of $f m$ illustrated by Connolly [1950] are represented in our sample, which also includes additional patterns. Lulu (online suppl. Fig. S8) manifests the simplest form of $f m$ in our sample (similar to Fig. 82 of Connolly [1950]), in which $\mathrm{fm}$ is connected to $h$ and $r$ in both hemispheres. Several of the hemispheres have sulcal patterns involving $\mathrm{fm}$ that are more complex than those illustrated by Connolly [1950]. For example, the left hemisphere of J. Carter (online suppl. Fig. S5) has a relatively long $f m$ that is independent of $r$ and $h$ and courses in a rostrocaudal direction that approximately parallels the dorsal perimeter of the frontal lobe when viewed laterally. A dorsomedially coursing sulcus near its caudal end connects $f m$ with $f s$ and there is an additional short fragment of $f m$ attached to $h$. Both hemispheres of Joseph (online suppl. Fig. S6) have long separate $f m s$ with that on the right coursing lateral and approximately parallel to a substantial superior frontal sulcus $(f s)$. Joseph's right middle frontal gyrus looks especially convoluted. In addition to having a fragment of $f m$ attached to $h$, the right hemisphere of Laz (online suppl. Fig. S7) has fragments of $f m$ attached to the caudal ends of two relatively long fragments of $f$ s. This results in a pattern of two long (combined $f m / f s$ ) sulci that course approximately parallel to each other above a combined $\mathrm{h} / \mathrm{fm}$ sulcus. These newly identified configurations for $\mathrm{fm}$ show that variation in chimpanzee frontal lobes includes more complex midfrontal gyri than previously described [Connolly, 1950; Falk, 2014]. The implication of this finding for interpreting australopithecine endocasts is discussed below.

\section{Dorsolateral Occipital Cortex}

The Lunate Sulcus and Annectant Gyri. Lateral views (Fig. 3, 4) confirm the well-known fact that chimpanzee brains regularly manifest $L s$ that arc rostral to obliquely oriented lateral calcarine sulci $(l c)$ that divide the occipital lobe into two main parts, similar to the lateral division of human occipital lobes [Alves et al., 2012]. Comparison of the dorsal views of the 8 chimpanzee brains (Fig. 5) reveals substantial variation in the location of the dorsal part of $L$ relative to the A-P axis, with Callie's and Lulu's $L s$ located in relatively rostral positions (consistent with their lateral views) and, at the other extreme, Laz's $L$ located at a comparatively caudal location (also seen in lateral views). The locations of the medial parts of $L$ tend to be somewhat asymmetrical and are highly so for some individuals (Evelyne and Ick), as is the case for 1 of the 3 chimpanzees (Chuck) illustrated by Holloway et al. [2003], in which the right hemisphere has a more posterior placement of $L$ compared to the left.

Connolly [1950] observed that caudal displacement of $L$ (when it occurs) is related to the superficial emergence of part or all of the first annectant gyrus of Gratiolet (1) that is usually buried within the depths of $L$ in most nonhuman anthropoids [Gratiolet, 1854; Connolly, 1950, p. 96; Iaria and Petrides, 2007]. This gyrus curves around the
Falk/Zollikofer/Ponce de León/ Semendeferi/Alatorre Warren/Hopkins 
parieto-occipital sulcus ( $p o$ ), bridges between the parietal and occipital lobes, and is usually exposed to some degree on the surfaces of chimpanzee brains, "though its posterior limb is quite narrow and may be wholly operculated" [Connolly, 1950, p. 111]. The 8 brains described here are consistent with the observation of Connolly [1950] that 1 is usually present but is operculated to varying degrees. The fullest exposure of 1 in our material is in Evelyne's left hemisphere (Fig. 5) in which the anterior portion of an Sshaped 1 curves around the lateral end of po and a substantial posterior limb of 1 displaces $L$ caudally compared to the $L$ on the right. More often, just the anterior limb of 1 is exposed and the posterior limb dips under the occipital operculum (Fig. 5, Abby, Callie, J. Carter, left hemisphere of Lulu). In Lulu's right hemisphere, on the other hand, 1 is fully operculated by $L$. Asymmetries in the positions of the medial ends of $L$ seen in the dorsal views of Evelyne and Lulu's brains (Fig. 5) lend credence to the observation of Connolly [1950, p. 96] that the emergence of 1 results in a "backward push" of $L$. This is also the case for the chimpanzee Chuck, in which the right $L$ appears to be pushed caudally compared to the left $L$ by an exposed (but unlabeled) 1 [Holloway et al., 2003, p. 597, Fig. 3].

Connolly [1950, p. 232] noted that in anthropoid apes, "part of the second transitional gyrus may also be exposed on the surface", which is the case bilaterally for Evelyne, Iyk, and Joseph (Fig. 5). As described and illustrated by Cunningham [1892, pp. 225-226], the second annectant gyrus of Gratiolet (2) in chimpanzees bridges between the angular gyrus near the middle ramus (a2) of the superior temporal sulcus $(t s)$ and the occipital lobe caudolateral to 1 . This gyrus is usually located within the depths of $L$, although portions may be exposed on the surface, in which case they are delimited by the medial and lateral branches of the transverse occipital sulci (otr) and $L$ [see Cunningham, 1890, plate IX, Fig. 2, and Benham and Oxoii, 1895, pp. $67-68$, plate 10 , Fig. 32 ]. $L$ frequently operculates 2 in chimpanzees, in which case it is not visible on the surface. Asymmetry in the sizes of 2 in Iyk (Fig. 5) suggests that a large exposure of this gyrus on the brain's surface may contribute (along with a relatively large exposure of $1 \mathrm{such}$ as that seen in Evelyne) to caudal displacement of $L$. The most dramatic representations of 2 in our sample are those on both of Joseph's hemispheres (Fig. 5).

Interestingly, Laz's brain, which has substantial $1 s$ bilaterally, also has additional exposed triangular patches of cortex bilaterally between the medial branches of otr and $L$ (covered by the medial labels for $L$ in Laz's brain, Fig. 5), similar to those described (but not illustrated) for gorillas by Connolly [1950, p. 104]. Connolly did not characterize this feature as part of an annectant gyrus (instead describing it as "a triangular area slightly depressed below the level of the neighboring cortex") and it is not clear whether Laz's are medial extensions of 2 (a possibility suggested by the right hemisphere of Iyk, Fig. 5) or simply superficially exposed parts of the cuneus [Bruner et al., 2017]. As noted, Laz's $L s$ appear to be located relatively caudal compared to the other chimpanzees' in Fig. 5, which may be associated with a caudal push (to paraphrase Connolly [1950]) from the combined exposure of these triangular patches and 1s. Two other features are notable on Laz's brain. Connolly [1950, p. 96] hypothesized that caudally displaced $L s$ due to the emergence of 1 "causes a crowding together of the (occipital) sulci, with modifications of their form", which seems to be the case for Laz's left hemisphere (Fig. 3, 5). (Note also the high degree of convolutedness of Joseph's occipital lobes, which may be associated with the combined effects of 1 and 2.) The second interesting feature in Laz (which may be seen more clearly by magnifying the dorsal images of his brain) is an apparent rupture in the opercular lip of the left $L$. Recalling that these images were obtained in vivo, it is not clear if this rupture was pathological or a natural variation associated with breaking up of the occipital operculum (i.e., that operculates $L$ ) in conjunction with caudal displacement of $L$.

Both of the annectant gyri ("folds" 1 and 2) described above for chimpanzees are fully exposed in human brains. Thus, "the superior parietooccipital fold, which encircles the external perpendicular fissure $(p o) \ldots$ [and] the inferior parieitooccipital fold, which is constituted by a posterior extension of the angular gyrus that converges with the superior occipital gyrus or with the middle occipital gyrus," were identified in $100 \%$ of human hemispheres examined by Alves, et al. [2012, p. 1018]. Chimpanzees have the same connecting gyri except that they tend to be operculated by $L$. As observed long ago in reference to these two gyri in chimpanzees: "If we now suppose the operculum abolished, and the two annectant gyri in relation to the intraparietal sulcus raised to the surface, we have the condition present in Man" [Cunningham, 1890, p. 150].

Implications of the Newly Described Chimpanzee Brains for Hominin Endocasts. The in vivo sulcal patterns described above for 8 chimpanzee brains include variations that have not been recognized previously in chimpanzees, some of which have implications for identifying and interpreting sulci reproduced on australopithecine and other small-brained hominin endocasts. Beginning with the frontal lobes, the suggestion that the anterior inferior frontal gyrus reproduced on the left hemisphere of the endocast from MH1 (A. sediba) aligns its "orbitofron- 
tal shape... most closely with humans endocasts" [Carlson et al., 2011, p. 1402] because it "displays a distinct ventrolateral bulge" [Carlson et al., 2011, pp. 1403, 1405, shaded feature F in their Fig. 2] is questionable in light of the fact that numerous chimpanzees described and illustrated above (Fig. 3, 4) have inferior frontal gyri that are expanded rostral to fo and some even manifest extra fragments of $f i$ in that part of the brain (which MH1 does not). For example, the MH1 configuration of $f i, f o$, and the "bulge" rostral to that [see Falk, 2014, Fig. 5] appears remarkably similar to that in the left hemispheres of Evelyne and J. Carter (Fig. 3), and the left hemisphere of MH1 in this region is less complex than the same region in the right hemisphere of Lulu (Fig. 4). Similarly, the assertions that the orbital cap region on the frontal lobe of the $\mathrm{DH} 3$ (H. naledi) endocast "indicates a modern Homo-like frontal brain organization despite its small size" and that "a clear vertical ramus of the lateral fissure and its horizontal branch permits easy identification of a modern configuration of the frontal opercula" [Hurst et al., 2017, p. 225] need reassessment in light of the range of variation in the inferior frontal gyrus of chimpanzees documented here. (See, e.g., the triangular patch delimited rostrally and caudally by two small sulci at the superior end of $f_{0}$ in the left hemisphere of Callie [Fig. 3].)

Falk [2014] hypothesized that australopithecine frontal lobes were derived toward a human condition in their middle frontal gyri because endocasts of Taung, Sts 60, the No. 2 specimen from Sterkfontein (A. africanus), and MH1 (A. sediba) have separate branches of $f m$ that "are rare in ape brains but typical of human brains, and... in all four (australopiths), $f m$ is lateral to a long superior frontal sulcus $(f s)$ " [Falk, 2014, p. 8]. In light of the new evidence provided here this hypothesis can be falsified. Separate fragments of $\mathrm{fm}$ are present in the left hemispheres of Callie, Joseph, and Laz, (Fig. 3) and the right hemispheres of Abby and Joseph (Fig. 4). More to the point, $f m$ courses lateral and parallel to a long $f s$ in the right hemisphere of Joseph (online suppl. Fig. S6, compare the two sides with the dorsal view) and a long sulcus that appears to be a composite of $f m$ and $f s$ courses below and parallel to a long $f s$ and above a relatively long $f m$ that extends from $h$ in the right hemisphere of Laz (online suppl. Fig. S7, compare the three views), which suggests the sulcal pattern (and indeed the entire middle frontal gyrus) in this chimpanzee is more complex than comparable sulcal patterns of the australopiths that have, so far, been described.

Turning to the parietooccipital region, sulci in this region do not reproduce well on endocasts of chimpanzees
[Clark et al., 1936] or the ape-sized endocasts of australopithecines, which may help explain why the existence and location of $L$ for australopithecines has been a bone of contention since Dart [1925] first mistook the lambdoid suture of Taung for $L$ [Dart, 1925; Falk, 2009]. Most paleoneurologists now agree that $L$ is not visible on Taung and that "none of the other published australopithecine brain endocasts have a clearly discernible LS" [Holloway et al., 2004b, p. 290], although one australopith endocast, i.e., that of Stw 505, remains controversial [Holloway et al., 2004b; see Falk, 2014, pp. 3-4, for a summary and figure that illustrates the conflicting sulcal identifications]. Although it is now known that, contrary to what was reported by Smith [1903], human ancestors lost $L$ at some point during hominin brain evolution [Allen et al., 2006], so far the fossil record of endocasts has been mute on exactly how and when this happened. Was a crescentshaped $L$ pushed caudally by expanding parietooccipital regions, possibly in conjunction with bulging of the parietal region and expansion of the medially located precuneus [Bruner et al., 2017], over an extended period of evolution until it reached a point near the occipital pole and then disappeared? Or did $L$ lose its opercular lid and disappear during an earlier period of hominin evolution as 1 and 2 became fully exposed (recall Laz)? Hopefully, a more complete fossil record of hominin endocasts (perhaps with larger brain volumes than the ape-sized endocasts of australopiths) will allow future paleoneurologists to answer this question.

Meanwhile, the variations in the locations of $L$ and their relationship to exposed portions of gyri that bridge between the parietal and occipital lobes ( 1 and 2) documented here for chimpanzee brains should be considered when describing and interpreting chimpanzee sulcal patterns and their implications for interpreting hominin endocasts [Holloway et al., 2003], including those from the newly named $H$. naledi [Holloway et al., 2017]. As is true for frontal lobe sulcal patterns, if a sulcal pattern reproduced in the parietooccipital region of an early hominin endocast is also represented among extant chimpanzees, it should not be interpreted as derived (relative to apes) toward a human condition.

\section{Conclusion}

As illustrated in Figure 1, both the frontal and occipital lobes in chimpanzees consistently manifest marked crescent-shaped sulci that are not present on the surfaces of human brains - fo and $L$, respectively. It is generally ac-
56

Brain Behav Evol 2018;91:45-58 DOI: $10.1159 / 000487248$
Falk/Zollikofer/Ponce de León/ Semendeferi/Alatorre Warren/Hopkins 
cepted that the former was displaced caudally (beneath the brain's surface) in conjunction with the evolutionary emergence of derived sulci in humans in the region of Broca's area, which is involved in speech production in the left hemisphere. It is also hypothesized that $L$ was displaced caudally during hominin evolution, and recent evidence confirms that its "true" homologue eventually disappeared [Allen et al., 2006; Alves et al., 2012]. The newly described sulcal variations described above for chimpanzees suggest that, among other factors [Bruner et al., 2017], $L$ may have been lost in conjunction with the full emergence of two annectant gyri (1 and 2), one of which entailed expansion of the angular gyrus (BA 39) - a region that is also important for human language. Significantly, the new sulcal variations reported here for both frontal and parietooccipital regions in chimpanzee brains are consistent with comparative diffusion tensor imaging findings from studies of a white-matter tract, the arcuate fasciculus, which arches around the Sylvian fissure linking temporal and frontal lobe areas. In contrast to humans, the arcuate fasciculus of chimpanzees is relatively more connected with the inferior parietal lobule, including the angular gyrus, than the temporal lobe [Rilling et al., 2008]. A more prominent temporal lobe projection of the arcuate fasciculus in humans "supports the transmission of word-meaning information stored in the MTG (middle temporal gyrus) and angular gyrus to pars triangularis and orbitalis for both sentence comprehension and sentence construction during spontaneous speech" [Rilling et al., 2008, p. 3]. Though speculative, this comparative research on the arcuate fasciculus combined with the variations documented above for chimpanzee sulci raises the possibility that language evolution may have been a primary factor in a coordinated emergence of the cortical sulcal patterns in the frontal and parietooccipital regions that set human brains apart from those of other higher primates (Fig. 1).

Apart from providing in vivo sulcal identifications for 16 chimpanzee hemispheres (which more than triples the number of hemispheres illustrated by Connolly [1950] [Fig. 2]), we also provide detailed information on patterns of interindividual variation. Online supplementary Figures S1-S8 offer a unique set of illustrations with comprehensive sulcal identifications for in vivo chimpanzee brains viewed from three aspects. Frontal lobe sulcal configurations that were previously believed to be derived in australopithecines (compared to apes) are represented in some of the newly described chimpanzee brains, and this finding overturns certain published hypotheses about frontal lobe evolution in small-brained hominins. Newly described variations in the superficial representation of two gyri that connect parietal and occipital cortices in chimpanzees and humans contribute to hypotheses about the specific mechanisms that may have been involved in evolutionary reorganization of the caudal part of the brain [Bruner et al., 2017]. Although it is beyond the scope of the present paper, future researchers who apply geometric morphometric approaches to the study of chimpanzee sulci [e.g., Gómez-Robles et al., 2015; Bruner et al., 2017] or wish to construct probability maps that illustrate variation in the locations of various sulci for chimpanzees [e.g., Schenker et al., 2010], as has been done for humans [Iaria and Petrides, 2007], will, of course, require accurate identifications of sulci as an initial step and should, thus, find the above descriptions useful. Investigators may also request MRI data for additional chimpanzee brains from the National Chimpanzee Brain Resource (http://www.chimpanzeebrain.org/). In sum, it is our hope that the above illustrations, identifications, and discussions of chimpanzee in vivo sulcal patterns will be useful for future researchers who attempt to decipher hominin endocasts to learn how and when the external morphology of human cerebral cortices evolved from apelike precursors.

\section{Acknowledgments}

This research was supported by NIH grants NS-42867, NS73134, and NS-92988 (W.D.H.) and Swiss National Science Foundation grant No. 31003A_135470/1 (C.P.E.Z.). Financial support was also provided by the School for Advanced Research in Santa Fe, NM, USA (D.F.). Dina Davis is thanked for preparation of illustrations, and we are grateful to two anonymous reviewers for helpful suggestions.

\section{Disclosure Statement}

The authors declare no conflict of interests.

References

Allen JS, Bruss J, Damasio H (2006): Looking for the lunate sulcus: a magnetic resonance imaging study in modern humans. Anat Rec A Discov Mol Cell Evol Biol 288:867-876.

Alves RV, Ribas GC, Parraga RG, de Oliveira E (2012): The occipital lobe convexity sulci and gyri. J Neurosurg 116:1014-1023.

Amunts K, Malikovic A, Mohlberg H, Schormann T, Zilles K (2000): Brodmann's areas 17 and 18 brought into stereotaxic space: where and how variable? Neuroimage 11:66-84.

Amunts K, Schleicher A, Burgel U, Mohlberg H, Uylings HB, Zilles K (1999): Broca's region revisited: cytoarchitecture and intersubject variability. J Comp Neurol 412:319-341. 
Bailey P, von Bonin G, McCulloch WS (1950): The Isocortex of the Chimpanzee. Urbana, University of Illinois Press.

Beaudet A, Dumoncel J, de Beer F, Duployer B, Durrleman S, Gilissen E, Hoffman J, Tenailleau C, Thackeray JF, Braga J (2016): Morphoarchitectural variation in South African fossil cercopithecoid endocasts. J Hum Evol 101: 65-78.

Benham WB, Oxoii HM (1895): A description of the cerebral convolutions of the chimpanzee known as "Sally", with notes on the convolutions of other chimpanzees and of two orangs. Q J Microsc Sci 37:47-86.

Bruner E, Preuss TM, Chen X, Rilling JK (2017) Evidence for expansion of the precuneus in human evolution. Brain Struct Funct 222: 1053-1060.

Carlson KJ, Stout D, Jashashvili T, de Ruiter DJ, Tafforeau P, Carlson K, Berger LR (2011): The endocast of MH1, Australopithecus sediba. Science 333:1402-1407.

Clark WEL, Cooper DM, Zuckerman S (1936): The endocranial cast of the chimpanzee. J R Anthropol Inst 66:249-268.

Connolly JC (1950): External Morphology of the Primate Brain. Springfield, Thomas.

Cunningham D (1890): The intraparietal sulcus of the brain. Trans R Acad Med Irel 8:495515.

Cunningham DJ (1892): Contribution to the Surface Anatomy of the Cerebral Hemispheres. Dublin, Academy House.

Dart RA (1925) Australopithecus africansus: the man-ape of South Africa. Nature 115:195199.

Duvernoy HM, Bourqouin P, Cabanis EA, Cattin F, Maeder P (1999): The Human Brain: Surface, Blood Supply, and Three-Dimensional Sectional Anatomy. New York, Springer.

Falk D (1978): External neuroanatomy of Old World monkeys (Cercopithecoidea). Contrib Primatol 15:1-95.

Falk D (2004): Hominin brain evolution: new century, new directions. Coll Antropol 28 (suppl 2):59-64.

Falk D (2009): The natural endocast of Taung (Australopithecus africanus): insights from the unpublished papers of Raymond Arthur Dart. Yearb Phys Anthropol 52:49-65.

Falk D (2014): Interpreting sulci on hominin endocasts: old hypotheses and new findings. Front Hum Neurosci 8:134.

Ferrier D (1886): The Functions of the Brain. New York, Putnam.

Gilissen E, Zilles K (1996): The calcarine sulcus as an estimate of the total volume of human striate cortex: a morphometric study of reliability and intersubject variability. J Hirnforsch 37: $57-66$.

Gómez-Robles A, Hopkins WD, Schapiro SJ, Sherwood CC (2015): Relaxed genetic control of cortical organization in human brains compared with chimpanzees. Proc Natl Acad Sci USA 112:14799-14804.

Gratiolet LP (1854): Mémoire sur les plis cérébraux de l'homme et des primatès: Avec un atlas (4 pp. XIV pl.) in fol. 33i. Paris, Bertrand. Holloway RL, Broadfield DC, Yuan MS (2003): Morphology and histology of chimpanzee primary visual striate cortex indicate that brain reorganization predated brain expansion in early hominid evolution. Anat Rec 273:594-602.

Holloway RL, Broadfield DC, Yuan MS (2004a): Brain Endocasts the Paleoneurological Evidence. Hoboken, Wiley, vol 3.

Holloway RL, Clarke RJ, Tobias PV (2004b): Posterior lunate sulcus in Australopithecus africanus: was Dart right? C R Palevol 3:287293.

Holloway RL, Hurst SD, Garvin HM, Schoenemann T, Vanti WB, Hawks J, Berger LR (2017) Homo naledi posterior endocasts and their significance for understanding brain organization. Am J Phys Anthropol 162:220.

Hurst SD, Holloway RL, Garvin HM, Schoenemann T, Vanti WB, Hawks J, Berger L (2017): Homo naledi's frontal lobe. Am J Phys Anthropol 162:225.

Iaria G, Fox CJ, Waite CT, Aharon I, Barton JJ (2008) The contribution of the fusiform gyrus and superior temporal sulcus in processing facial attractiveness: neuropsychological and neuroimaging evidence. Neuroscience 155: 409-422.

Iaria G, Petrides M (2007) Occipital sulci of the human brain: variability and probability maps. J Comp Neurol 501:243-259.

Jerison HJ (1973): Evolution of the Brain and Intelligence. New York, Academic Press.

Keller SS, Crow T, Foundas A, Amunts K, Roberts N (2009): Broca's area: nomenclature, anatomy, typology and asymmetry. Brain Lang 109: 29-48.

Mingazzini G (1928): Beitrag zur Morphologie der äusseren Grosshirnhemisphärenoberfläche bei den Anthropoiden (Schimpanse und Orang). Arch Psychiatr Nervenkr 85:1219.

Minh N Van, Hamada Y (2017): Age-related changes of sulcal imprints on the endocranium in the Japanese macaque (Macaca fuscata). Am J Phys Anthropol 163:285-294.

Ono M, Kubik S, Abernathy CD (1990) Atlas of the Cerebral Sulci. New York, Hafner.

Pearce E, Stringer C, Dunbar RI (2013): New insights into differences in brain organization between Neanderthals and anatomically modern humans. Proc Biol Sci 280:20130168.

Petrides M, Orr SL, Sprug-Much T (2011): The intermediate frontal sulcus and its relation to the superior frontal sulcus. https://www. researchgate.net/publication/280529896_ The_intermediate_frontal_sulcus_and_its relation_to_the_superior_frontal_sulcus (accessed August 19, 2017).

Petrides M, Tomaiuolo F, Yeterian EH, Pandya DN (2012): The prefrontal cortex: comparative architectonic organization in the human and the macaque monkey brains. Cortex 48: 46-57.

Rademacher J, Caviness Jr V, Steinmetz H, Galaburda A (1993): Topographical variation of the human primary cortices: implications for neuroimaging, brain mapping, and neurobiology. Cereb Cortex 3:313-329.

Radinsky LB (1972): Endocasts and studies of primate brain evolution; in Tuttle $\mathrm{R}$ (ed): The Functional and Evolutionary Biology of Primates. Chicago, Aldine, pp 175-184.

Rilling JK, Glasser MF, Preuss TM, Ma X, Zhao T, Hu X, Behrens TE (2008): The evolution of the arcuate fasciculus revealed with comparative DTI. Nat Neurosci 11:426.

Schenker NM, Hopkins WD, Spocter MA, Garrison AR, Stimpson CD, Erwin JM, Hof PR, Sherwood CC (2010): Broca's area homologue in chimpanzees (Pan troglodytes): probabilistic mapping, asymmetry, and comparison to humans. Cereb Cortex 20:730-742.

Segal E, Petrides M (2012): The morphology and variability of the caudal rami of the superior temporal sulcus. Eur J Neurosci 36:20352053.

Semendeferi K, Damasio H, Frank R, Van Hoesen GW (1997): The evolution of the frontal lobes: a volumetric analysis based on three-dimensional reconstructions of magnetic resonance scans of human and ape brains. J Hum Evol 32:375-388.

Semendeferi K, Teffer K, Buxhoeveden DP, Park MS, Bludau S, Amunts K, Travis K, Buckwalter J (2011): Spatial organization of neurons in the frontal pole sets humans apart from great apes. Cereb Cortex 21:1485-1497.

Sherwood CC, Broadfield DC, Holloway RL, Gannon PJ, Hof PR (2003): Variability of Broca's area homologue in African great apes: implications for language evolution. Anat Rec A Discov Mol Cell Evol Biol 271:276-285.

Sled JG, Zijdenbos AP, Evans AC (1998): A nonparametric method for automatic correction of intensity nonuniformity in MRI data. IEEE Trans Med Imaging 17:87-97.

Smith GE (1903): The so-called Affenspalte in the human (Egyptian) brain. Anat Anz 24:74-83.

Tallinen T, Chung JY, Rousseau F, Girard N, Lefèvre J, Mahadevan L (2016): On the growth and form of cortical convolutions. Nat Phys 12:588-593.

Tustison NJ, Avants BB, Cook PA, Zheng Y, Egan A, Yushkevich PA, Gee JC (2010): N4ITK: improved N3 bias correction. IEEE Trans Med Imaging 29:1310-1320.

Van Essen DC (1997): A tension-based theory of morphogenesis and compact wiring in the central nervous system. Nature 385:313-318.

Von Bonin G (1949): Architecture of the precentral motor cortex and some adjacent areas; in Bucy PC (ed): The Precentral Motor Cortex. Urbana, University Illinois Press, pp 7-82.

Wang X, Studholme C, Grigsby PL, Frias AE, Cuzon Carlson VC, Kroenke CD (2017): Folding, but not surface area expansion, is associated with cellular morphological maturation in the fetal cerebral cortex. J Neurosci 37: 1971-1983.

Walker AE, Fulton JF (1936): The external configuration of the cerebral hemispheres of the chimpanzee. J Anat 71:105-116. 